\title{
Improvement of Dynamic Stability of a Single Machine Infinite-Bus Power System using MPC based Power System Stabilizer
}

\author{
Mohamed M. Ismail ${ }^{1}$, Ahmed F. Bendary ${ }^{1}$ \\ ${ }^{1}$ Department of Electrical Eng., Helwan University, Cairo, Egypt.

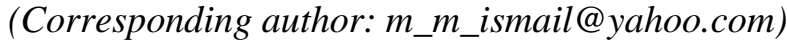

\begin{abstract}
The damping during low frequency oscillation is one of a troublesome problem that occurs in single machine infinite-bus power system, power system stabilizers (PSSs) are used to solve this problem. In This paper, a study of using fuzzy logic technique to enhance the stability of a single machine infinite bus system is proposed. Fuzzy logic control is used to design the control system of PSSs to achieve recognized improvement in Dynamic Stability. The usage of this technique which based on Artificial Intelligence (AI) fuzzy logic and Model Predictive Control (MPC) made the signal damped out very fast, reduced Steady state error, maximum overshooting.
\end{abstract}

Keywords: Induction motor drive; energy saving; reducing voltage; motor losses.

\section{Introduction}

Power system stability is a property of a power system that enables it to remain in a state of operating equilibrium under normal operating conditions. Small signal and transient are two Factors that influence on the stability of the system. Small signal stability is the ability of the system to return to a normal operating state following a small disturbance or gradually loading. Transient stability is the ability of the system to return to a normal operating state following a severe disturbance, such as a single or multi-phase short circuit or a generator loss. Low frequency oscillations are a major problem in large power systems. A power system stabilizer provides the supplementary control signal to the excitation system of the electric generating unit for damping these low frequency oscillations. Power system stabilizers are successfully used in power systems for few years because of their flexibility low cost and easy implementation. The power system stabilizer is used to generate supplementary control signals to damp the low frequency oscillation. The conventional power system stabilizer was widely used in existing power systems and has contributed to the enhancement of the dynamic stability of power systems [1]. The parameters of conventional power system stabilizer are based on linearized model of power system around of nominal operating point. Power systems are highly nonlinear systems so the design of conventional power system stabilizer based on linearized model of the power systems cannot guarantee its performance in practical operating environment as it faces many problems concerning its design to give fixed desired damping at a fixed operating point which is defined by the terminal voltage as well as active and reactive power, with the change in operating point many PSS

based on classical theory are not able to give desired performance [2].To improve the performance of conventional power system stabilizer and the pre mentioned problems many techniques have been proposed for the design of robust control system using many types of AI techniques like: genetic algorithm, Particle swarm neural network, simulated annealing fuzzy logic $[3,4]$ and many other intelligent optimization techniques like Bat algorithm (BA) which used in design of a proportional, derivative, and integral (PID) based power system stabilizer (PSS) to enhance the small signal stability of nonlinear power system for a wide range of operating conditions [5]. Also, the Discrete PID controller was used instead of Conventional Proportional Integral Derivative (PID) with PSS system to give improvement for the stability of multi-machine system in SIMULINK environment [6]. Model predictive control (MPC) refers to a class of computer control algorithms that utilize an explicit process model to predict the future response of plant [9-15]. Figure 1 shows the block diagram of the MPC. It is designed based on multi-step optimization objectives. Future plant responses are predicted using an identified model developed earlier for processes under control and optimized at regular intervals using the trajectory of manipulated inputs with respect to output reference and performance index for optimization process. MPC algorithms can control complex systems with multi-control variables and provides an inherently systematic approach dealing 
with constraints on inputs and states. There are number of key elements and concepts that together form what is called model predictive controller prediction model as (Objective function, Receding control horizon and Constraints). There are multiple formulas to define the optimization problem of MPC, which commonly addressed as objective function. The typical formula is the quadratic programming (QP) formulation, where the objective function is quadratic and uses linear constraints. Another form of objective function is linear programming (LP) form; this expresses both the objective and constraints linearly. According to [1] this method provides smoother control actions for MPC. This work uses the quadratic programming format.Ref.[16] made Nonlinear dynamic analysis of a single-machine infinite-bus power system. The simulations are implemented using MATLAB - SIMULINK as a simulation program tool.

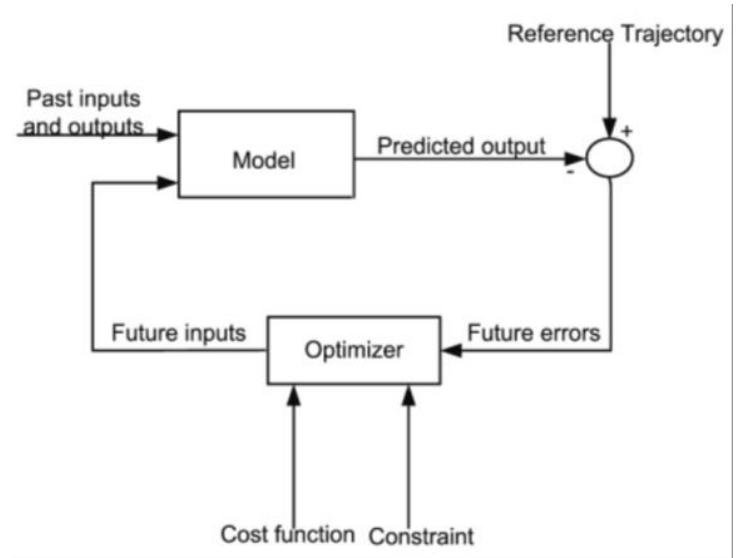

Figure (1): Model Predictive Control (MPC) Concept [17]

\section{System under Study}

One of the most important equipment's used in electric power systems is synchronous Machine as it considers as an important source of electric energy. The system under study consists of synchronous machine, excitation system and power system stabilizer, where the synchronous generator deliver electric power to the infinite bus through a single circuit transmission line, Eg and EB are the generator terminal and infinite bus voltage respectively as shown in Figure (2). Table (1) shows the parameters of the system under study [7].

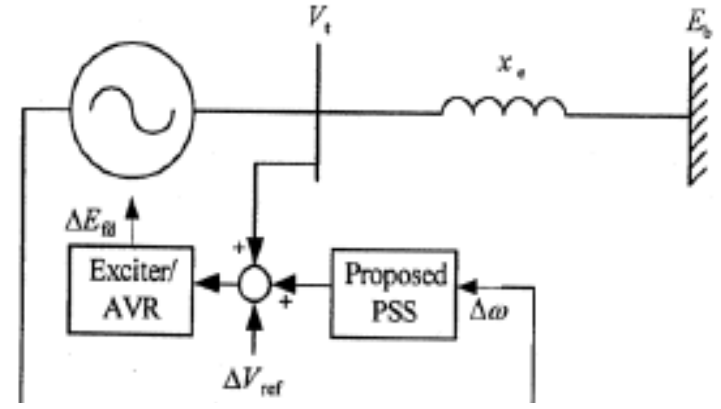

Figure (2): Synchronous Machine Connected to Infinite Bus

Table (1) The parameters of the system under study

\begin{tabular}{|l|l|}
\hline Inertia Constant, $\mathrm{H}$ & s/KVA-KW2.37 \\
\hline Generator Rating & MVA 160 \\
\hline Rated Voltage & $\begin{array}{l}\text { 'KV 15 } \\
\text { Connected-Y }\end{array}$ \\
\hline Voltage Excitation & KV 375 \\
\hline${ }^{\text {G }}$ Stator Current, I & A 6158.4 \\
\hline Field Current & A 926 \\
\hline Power factor & 0.85 \\
\hline${ }^{\text {d}} \mathrm{X}$ & 1.7 \\
\hline${ }^{\mathrm{d}} \mathrm{X}$ & 0.245 \\
\hline${ }^{\mathrm{q}} \mathrm{X}$ & 1.64 \\
\hline 'Xq & 0.38 \\
\hline${ }^{\mathrm{s}} \mathrm{R}$ & 0.001096 \\
\hline${ }^{\text {do }} \mathrm{T}$ & 5.9 \\
\hline
\end{tabular}

\section{Problem Formulation}

In this section, the power system stability problem is formalized and presented through modeling the single machine infinite bus system using MATLAB/Simulink tools, the modeling of this system was presented in detail in [8]. The lack of synchronizing or damping torque due to small disturbances lead to a steady increase or decrease in rotor angle so the input signal of the proposed controller will be the speed deviation and the output signal is the frequency signal. In this paper, five different cases for the proposed PSS are presented, the first case shows the PSS installed in Single Machine Infinite-Bus Power System without using any type of control techniques, the second case presents the PSS response when using PID controller, the third case presents PSS response when use PSS based on PID tuned by Fuzzy Logic, the fourth case presents PSS based on PID and MPC while the fifth case presents PSS based on PID tuned by Fuzzy Logic with the help Model Predictive 
Control, in order to show the effects of these controllers and how much the enhancement in the output signal is achieved.

i) Case (1): SMIB with PSS without Controller (Base case)

The power system stabilizer can help to damp generator rotor oscillations by providing an additional input signal that produces a torque component in phase with the speed deviation. The input signal may be rotor speed, real power, frequency, or some other signal. The output signal is passed to the AVR as auxiliary signal. The parameters within the PSS are carefully selected for each PSS depending on its input signals and location in the system The input signal is the speed deviation signal, and the output signal is the frequency signal. Figure (3) shows modeling of PSS without the help of the proposed controllers using MATLAB/ Simulink tools, the blocked diagram consists of gain washout block and two stage lead-lag block.

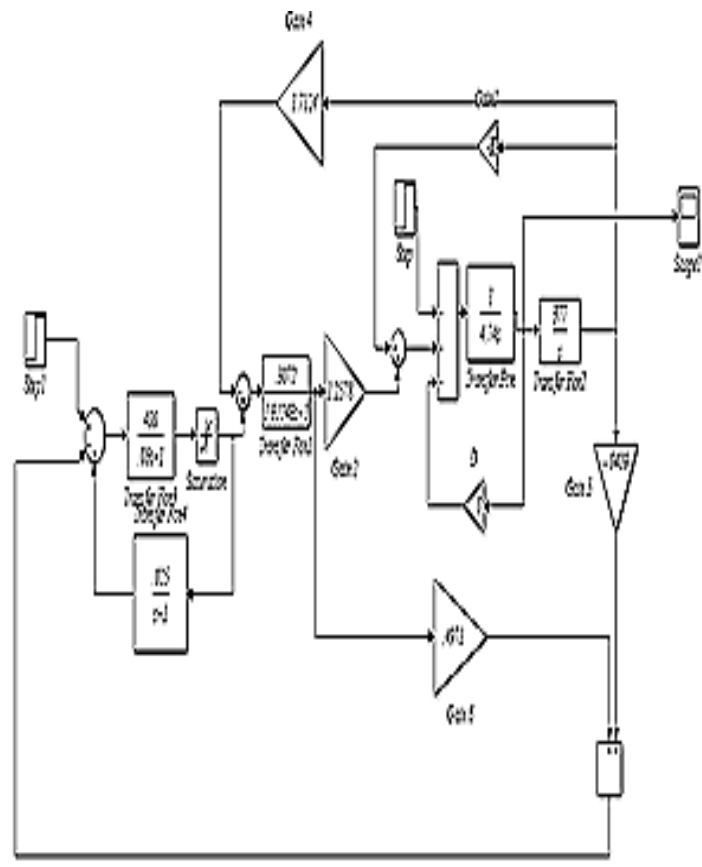

Figure (3) Block Diagram of PSS System without Controller

ii) Case (2): SMIB with PSS based on PID Controller:

This session presents the effects of adding PID controller to the SMIB system connected to PSS. Figure (4) shows the modeling of PSS with the help of PID controllers, the value of proportional, derivative and integral gain is taken according to Ziegler and Nichols method.

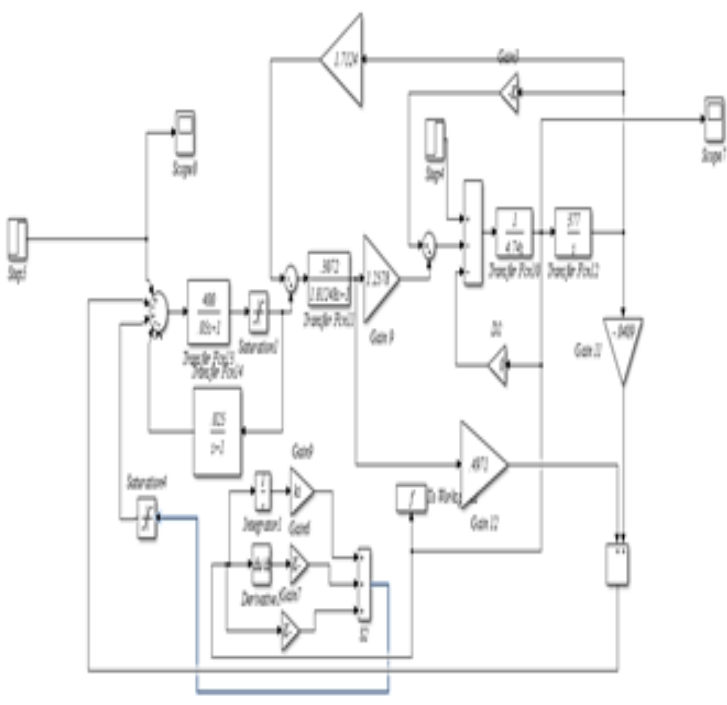

Figure (4) PSS based on PID Controller

iii) Case (3): SMIB with PSS based on PID tuned by Fuzzy Logic:

Fuzzy logic provides one of the most effective alternative AI techniques for stability enhancement. The proposed method using fuzzy logic showed better improvement than conventional power system stabilizer. Fuzzy logic rules were developed for triangular membership function of input and output variables. Figure (5) shows the SMIB with PSS Controller based on fuzzy logic.

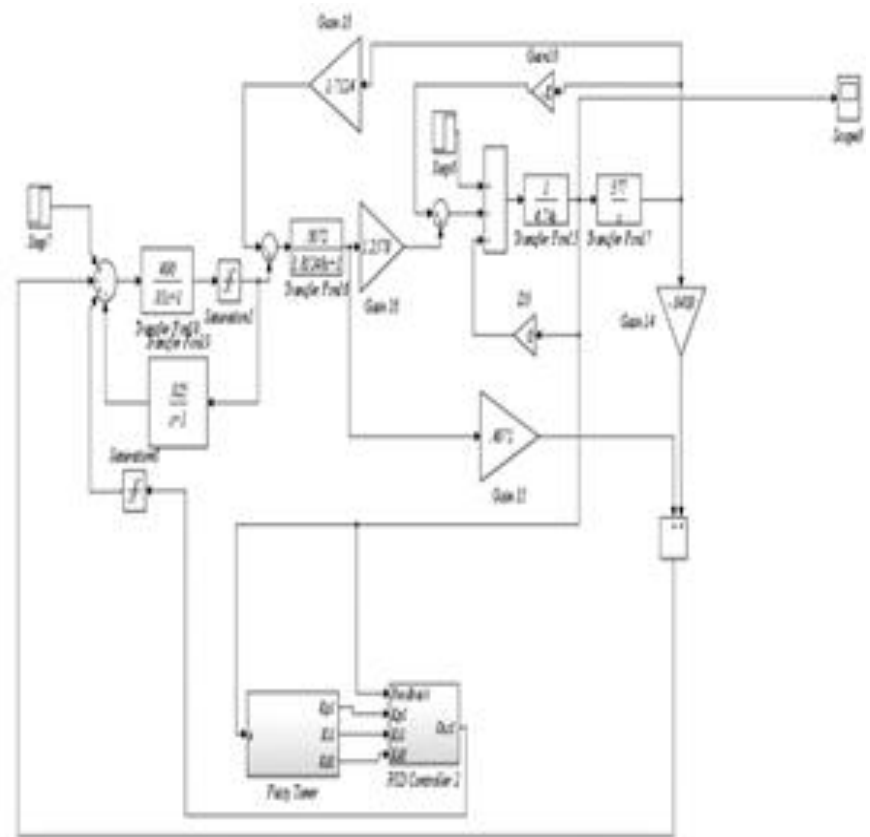

Figure (5) presents the SMIB with PSS Controller based on fuzzy logic 
iv) $\quad$ Case (4) SMIB with PSS based on PID and MPC:

Model Predictive Control (MPC) is considered one of the newest control techniques appears as a solution of the problems faced by the conventional PID controller when the Processes are difficult to control with standard PID algorithm - long time constants, substantial time delays, inverse response, and there is substantial dynamic interaction among controls, i.e., more than one manipulated variable has a significant effect on an important process variable. Figure (6) shows the SMIB with PSS Controller based on MPC controller.

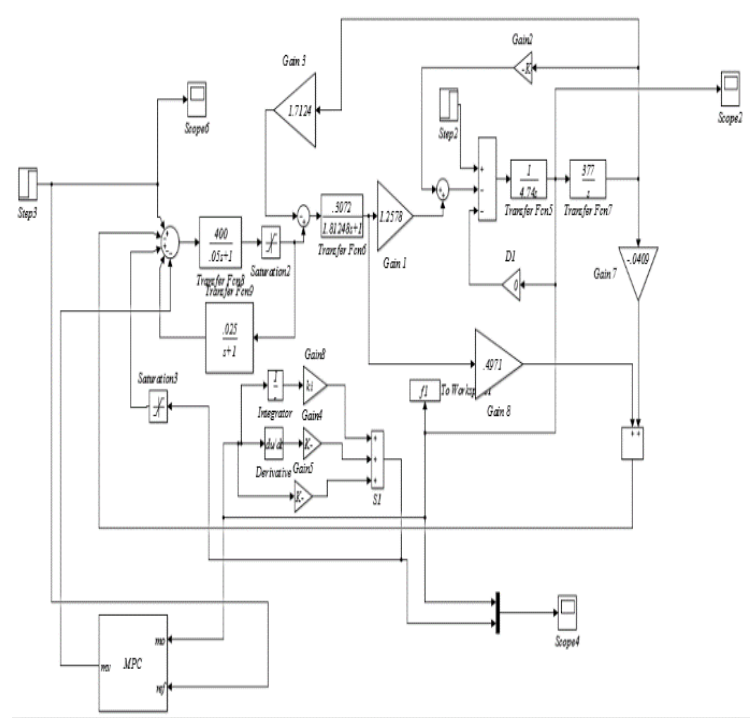

Figure (6) presents a block diagram for PSS controller based on MPC

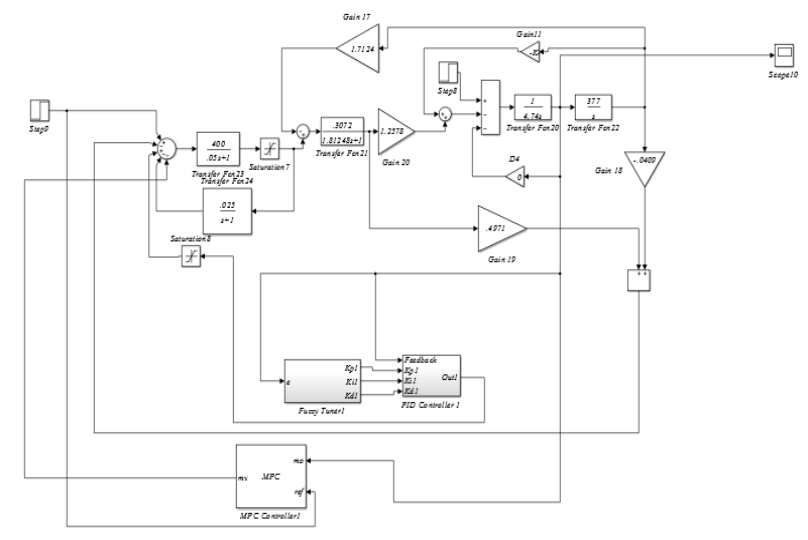

v) Case (5) SMIB PSS based on PID tuned by

Figure (7) Block Diagram of PSS Controller Based on Fuzzy and MPC Fuzzy Logic with the help of Model Predictive Control
The simulation is repeated by using a combination between MPC and fuzzy logic controller. Figure (7) shows the SMIB with PSS Controller based on MPC and fuzzy logic controller.

\section{Simulation Results}

This section presents the simulation results of the studied system through showing the performance of the proposed algorithms has been studied by means of MATLAB simulation. Figure (8) represents the enhancement in the output signal response of frequency signal versus time compared to base case when using PID controller. With the PID based controller, oscillations are damped out very fast in less than $2.5 \mathrm{sec}$. than as compared to when without controller. The steady state error is decreased as can be observed from Figure (8). Also, the overshoot in these states is less without the controller.

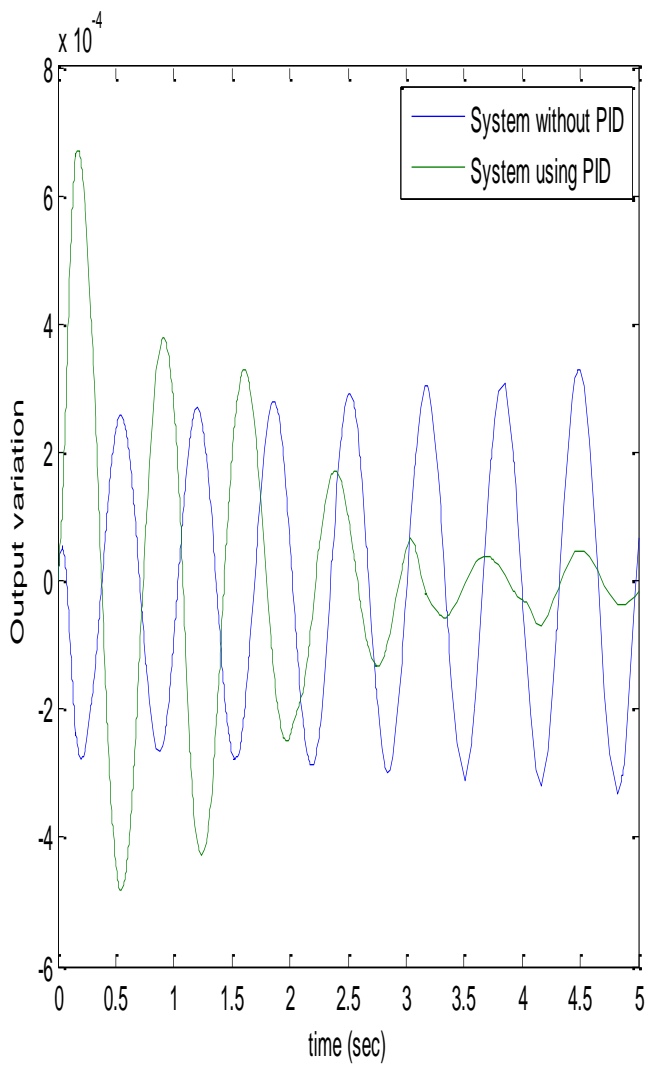

Figure (8) represents the in the output signal response of frequency signal versus time compared to base case

Figure (9) shows the output response of frequency and voltage signal versus time after adding both PID and MPC. With both PID based controller and MPC, oscillations are damped out very fast in less than 1 sec. than as compared to previous cases. Also, the steady state error is decreased as can be observed 
from Figure (9). With the PID based controller and MPC, oscillations are damped out very fast in less than $1.5 \mathrm{sec}$. than as compared to when without controller. Also, the steady state error is decreased as can be obtained from Figure (9). Also, the overshoot in these states is also become more damped compared with the previous cases. Figure (10) shows the output response of frequency and voltage signal versus time after adding both fuzzy PID and MPC. With both fuzzy PID and MPC, oscillations are damped out very fast in less than $0.25 \mathrm{sec}$. than as compared to previous cases. Also, the steady state error is decreased as can be observed from Figure (10). the overshoot in these states is also decreased compared with the previous cases.

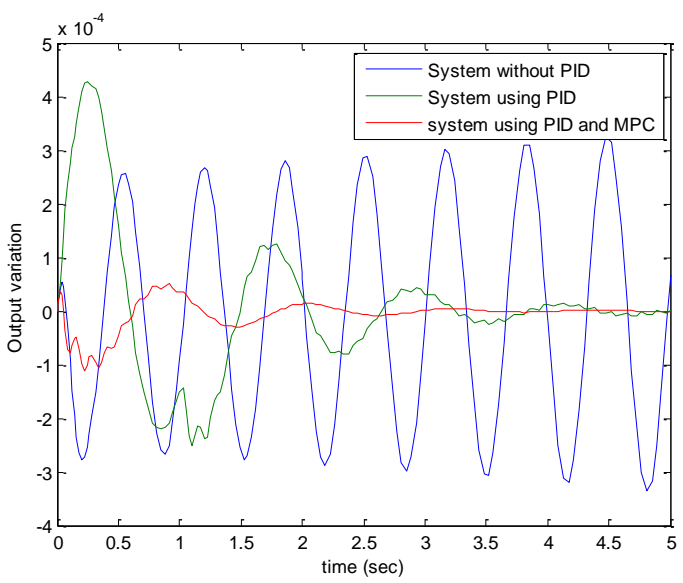

Figure (9) presents the output response of frequency and voltage signal versus time

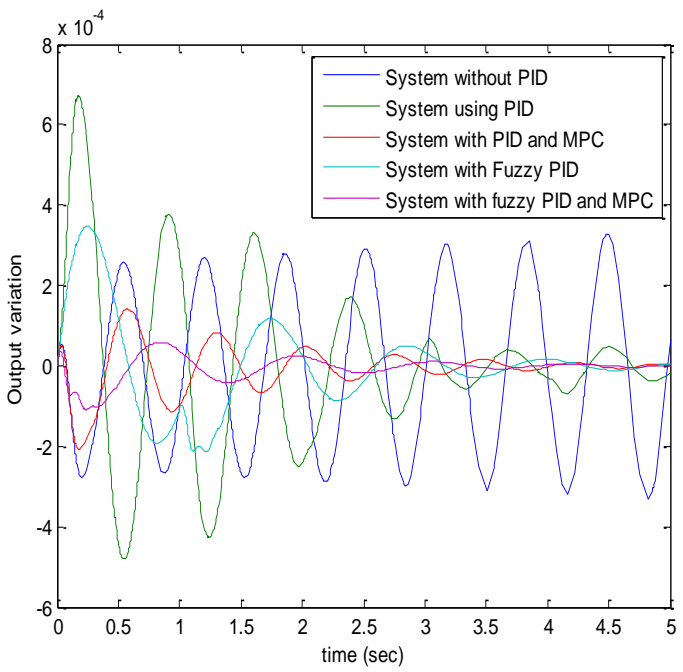

Figure (10) presents the output response of frequency and voltage signal versus time
Table (2) Comparative System Performance

\begin{tabular}{|l|c|c|}
\hline \multicolumn{1}{|c|}{ Controller Type } & $\begin{array}{c}\text { Steady } \\
\text { state error } \\
(10 \mathrm{e}-4)\end{array}$ & $\begin{array}{c}\text { Max } \\
\text { Overshoot }\end{array}$ \\
\hline $\begin{array}{l}\text { PSS without } \\
\text { Controller }\end{array}$ & 3.2 & 3 \\
\hline $\begin{array}{l}\text { PSS based on PID } \\
\text { Controller }\end{array}$ & 0.2 & 6.2 \\
\hline $\begin{array}{l}\text { PSS based on PID } \\
\text { tuned by Fuzzy } \\
\text { Logic }\end{array}$ & 0.18 & 3.8 \\
\hline $\begin{array}{l}\text { PSS based on PID } \\
\text { and MPC }\end{array}$ & 0.13 & 0.15 \\
\hline $\begin{array}{l}\text { PSS based on PID } \\
\text { tuned by Fuzzy } \\
\text { Logic with the help } \\
\text { of MPC }\end{array}$ & 0.05 & 0.2 \\
\hline
\end{tabular}

\section{CONCLUSIONS}

Small signal stability of a synchronous machine connected to an infinite bus has been improved using variable structure control. The results indicate that with the proposed VSC there is significant damping in the system. Also, great enhancement is achieved in signal output response. The PSS design using this approach can be implemented for large power systems which has many modes of low frequency oscillations. It was found that the PSS based on PID tuned by Fuzzy Logic with the help of MPC response outperforms is better than the other techniques used.

\section{REFERENCE}

[1] Vernekar, Pratik \& Wang, Zhongkui \& Serrani, Andrea \& Passino, Kevin. Feedback Control Methods for a Single Machine Infinite Bus System.

10.20944/preprints202009.0311.v1.),2020, PP. 53-59.

[2] Kour, Er \& Mehta, Shivinder," Power System Stabilizer for Single Machine Infinite Bus System".

10.15662/IJAREEIE.2017.0605060.,2017,pp.354 $2-3547$

[3] Kazmierkowski, M.P, 'Power System Dynamics: Stability and Control", Third Edition [Book News]. IEEE Industrial Electronics Magazine. $\begin{array}{lll}14 . \quad 94-95 . & 10.1109 / \mathrm{MIE} .2020 .2985200 .\end{array}$

[4] Garima Maheshwari and Nandkishore Meena. "Single Machine Infinite Bus System using GA and PSO"; International Journal of Digital 
Application \& Contemporary Research (IJDACR)Volume 4, Issue 6, January 2016),pp.67-70.

[5] Dhanesh K. Sambariya and Rajendra Prasad; "Design of Optimal Proportional Integral Derivative Based Power System Stabilizer Using Bat Algorithm"; Hindawi Publishing Corporation Applied Computational Intelligence and Soft Computing, Volume 2016, Article ID 8546108.

[6] Rakesh Kumar and Shailesh M. Deshmukh; "Stabilizing a Multi Machine Infinite Bus viaDiscrete PID Controller with PSS Excitation System", International Journal of Advanced Research in Electrical, Electronics and Instrumentation Engineering, Vol. 5, Issue 4, April 2016.

[7] Balwinder Singh Surjan, "Linearized Modeling of Single Machine Infinite Bus Power System and Controllers for Small Signal Stability Investigation and Enhancement", International Journal of Advanced Research in Computer Engineering \& Technology (IJARCET) Volume 1, Issue 8, October 2012.

[8] E. Kirankumar, Prof. V. C. Veera reddy and P. Hema chandu, "Stability enhancement of Power systems by tuning FACTS controllers", International Journal of Applied Engineering Research ISSN 0973-4562 Volume 11, Number 8 (2016) pp 5947-5955.

[9] Zanin, A. C., de Gouvea, M. T., \& Odloak, D. (2002). Integrating real-time optimization into the model predictive controller of the FCC system. Control Engineering Practice, 10(8), 819-831.

[10] Yüzgeç, U., Palazoglu, A., \& Romagnoli, J. A. (2010). Refinery scheduling of crude oil unloading, storage and processing using a model predictive control strategy. Computers \& Chemical Engineering, 34(10), 1671-1686.
[11] Amrit, R., "Optimizing process economics in model predictive control" (Doctoral dissertation, UNIVERSITY OF WISCONSINMADISON),2011.

[12] Xu, F., Huang, B., \& Tamayo, E. C.. "Assessment of economic performance of model predictive control through variance/constraint tuning. Proc IFAC ADCHEM", Gramado, Brazil,2006, 899-904.

[13] Maciejowski, J. M. (2002). Predictive control: with constraints. Pearson education.

[14] Qin, S. J., \& Badgwell, T. A. (2003). A survey of industrial model predictive control technology.Control engineering practice, 11(7), 733-764.

[15] Tyagunov, A. A. (2004). High-performance model predictive control for process industry. $\mathrm{PhD}$, Technische Universiteit Eindhoven, Rusland.

[16] Xiaodong Wang, Yushu Chen, Gang Han, Caiqin Song,"Nonlinear dynamic analysis of a single-machine infinite-bus power system",Applied Mathematical Modelling,Volume 39, Issues 10-11,2015,pp. 2951-2961,

[17] Derouiche, Mohamed \& Bouallègue, Soufiene \& Haggege, Joseph \& Sandou, Guillaume, "LabVIEW Perturbed Particle Swarm Optimization Based Approach for Model Predictive Control Tuning. IFAC-Papers" On Line. $49 . \quad 353-358$. 10.1016/j.ifacol.2016.07.138.,2016,pp.353-358. 